\title{
Zoonotic Soil-Transmitted Helminths in Free-Roaming Dogs, Kiribati
}

\author{
Patsy A. Zendejas-Heredia, Allison Crawley, Helen Byrnes, Rebecca J. Traub, Vito Colella
}

Soil-transmitted helminths are highly prevalent in the Asia-Pacific region. We report a $96.5 \%$ prevalence of zoonotic soil-transmitted helminths in dogs in Kiribati. We advocate for urgent implementation of treatment and prevention programs for these zoonotic pathogens, in line with the Kiribati-World Health Organization Cooperation Strategy 2018-2022.

Soil-transmitted helminths (STHs) are a group of $S$ parasitic worms infecting both humans and animals living in resource-limited settings (1). STHs affect $>2$ billion persons worldwide, causing major physical and cognitive impairment in children and negative health outcomes in pregnant women and women of childbearing age (2). Hookworms alone infect nearly half a billion persons, causing iron-deficiency anemia, stunted growth, and malnutrition (3). In addition, iron deficiencies may increase the risk of bacterial infections, especially in children $<5$ years of age (3). Although STHs are largely considered human-specific parasites, dogs are also known to harbor STHs that cause well-documented zoonotic diseases globally (3). The Ancylostoma ceylanicum roundworm is a zoonotic STH with dogs as reservoirs and is the second most common hookworm infecting humans in many regions in Southeast Asia and the Western Pacific (3). In humans, canine hookworms cause cutaneous larva migrans; A. braziliense hookworm is the only species capable of causing creeping eruptions and A. caninum hookworm triggers eosinophilic enteritis and aphthous ileitis (4). Recently, A. caninum eggs have been reported in the feces of human patients, suggesting that this parasite may

Author affiliations: University of Melbourne, Parkville, Victoria,

Australia (P. Zendejas-Heredia, R.J. Traub, V. Colella);

independent researcher, Brisbane, Queensland, Australia

(A. Crawley); Vets Beyond Borders, Brisbane (H. Byrnes)

DOI: https://doi.org/10.3201/eid2708.204900 complete its life cycle in humans, which can potentially result in disease transmission between hosts (4). In dogs, infections with hookworms are a common cause of hemorrhagic diarrhea and death in pups and chronic iron deficiency anemia in adult animals (4). The existence of dogs in close proximity to humans living in poor-hygiene settings, coupled with a lack of veterinary services and zoonotic awareness, exacerbates infection risks for the transmission of zoonotic STHs (5). In the Pacific islands, data on STH prevalence is scarce; therefore, estimates of disease burden caused by STHs cannot be accurately assessed.

Kiribati is a sovereign state in Micronesia in the central Pacific Ocean and is one of the most geographically isolated and impoverished countries in the world (6). Effects of poverty and climate change exert a huge toll on the ecology and health of humans and animals inhabiting the country. For instance, in the capital, South Tarawa, the high level of poverty, overcrowding, and presence of free-roaming animals influence the epidemiology of zoonotic STHs, trapping poor persons in a vicious cycle of poverty $(6,7)$. Despite the Kiribati-World Health Organization Cooperation Strategy 2018-2022 (6), to date, no information is available on the presence and diversity of zoonotic STHs in free-roaming animals in Kiribati.

\section{The Study}

The republic of Kiribati consists of 32 atolls in the central Pacific Ocean, with a population of $>110,000$ persons, inhabiting mainly the Gilbert Islands. The main economic revenue comes from seafood exports and fishing. Most primary foods are imported, and safe water supplies, proper solid waste disposal, and sanitation facilities are scarce, posing major threats to public health (6).

We investigated the occurrence of zoonotic STHs in free-roaming dogs in Tarawa Atoll, Kiribati, as part of a dog health and population management program 


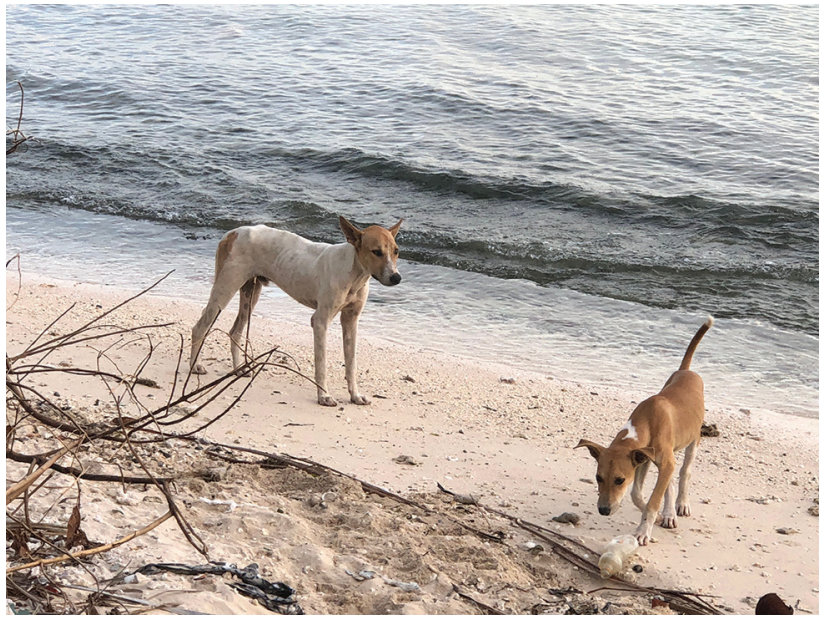

Figure 1. Living conditions of dogs on Tarawa Atoll, Kiribati.

led by the Mardi Chi Dingo Foundation (https:/ / farriervet.com/mardi-chi; Figure 1), which aims to seek a sustainable locally driven solution to improving animal health and overpopulation problems. The protocol of this study was approved by the Animal Ethics Committee at the Faculty of Veterinary and Agricultural Sciences (University of Melbourne, Melbourne, Victoria, Australia; ethics identification no. 1914930).

On the basis of previous surveys in regions with similar ecologic conditions $(\approx 80 \%$ expected prevalence of enteric parasites) (5), we estimated that $\approx 200$ dogs needed to be sampled in Kiribati to assess the prevalence of zoonotic STHs with 95\% confidence and to detect a pathogen, if present, at a prevalence of $\geq 0.5 \%$ (assuming diagnostic sensitivity of $80 \%$ and specificity of $98 \%$ ). To minimize animals' stress, we collected fecal samples from the rectal ampulla coinciding with the dog being anesthetized just before desexing surgery. We immediately preserved the fecal samples in Zymo DNA/RNA Shield (Zymo Research, https://www.zymoresearch.com), which renders any potential pathogen inactive or noninfective. We subjected fecal samples (200 mg each) from 198 dogs to genomic DNA extraction at the University of Melbourne using a Maxwell RSC PureFood GMO and Authentication Kit (Promega, https://www.promega.com) according to the manufacturer's instructions with modifications in that we performed an additional bead-beating step with $400 \mu \mathrm{L}$ CTAB buffer using $0.5 \mathrm{~mm}$ zirconia/silica beads (Daintree Scientific, http:/ / www.daintreescientific.com.au) using a FastPrep-24 5G Instrument (MP Biomedicals, https://www.mpbio.com). After bead-beating and cell lysis, we proceeded with DNA purification in a Maxwell RSC 48 Instrument (Promega). We stored the final eluted sample $(100 \mu \mathrm{L})$ at $-20^{\circ} \mathrm{C}$ for further analyses. We subjected the extracted DNA to multiplex quantitative PCR screenings for hookworm species (4) and Strongyloides stercoralis (8). We analyzed and visualized the data with GraphPad Prism version 8.0 (GraphPad Software, https://www.graphpad.com).

Overall, 96.5\% (95\% CI 93.9-99.0) of dogs were positive for $\geq 1$ of the investigated parasites. A total of $93.4 \%$ (95\% CI 92.5-98.4) were positive for $A$. caninum, $26.3 \%$ (95\% CI 20.1-32.4) for A. ceylanicum, $16.2 \%$ (95\% CI 11.5-21.9) for A. braziliense, and 29.8\% (95\% CI 23.4-361) for S. stercoralis (Figure 2).

\section{Conclusions}

We demonstrated that dogs play a major role in contaminating the environment with zoonotic STH species, potentially serving as reservoirs for infections of humans living in Kiribati. Current control strategies against STHs in Kiribati have been based on deworming of school-age children as part of the Pacific program to eliminate lymphatic filariasis using albendazole as prophylactic treatment (9). However, this drug has limited effects against $S$. stercoralis, which requires ivermectin for its effective control and for which public health strategies are yet to be developed $(10,11)$. Similarly, the emerging zoonotic agent $A$. ceylanicum has been reported with high prevalence, and despite $\approx 100$ million persons currently infected with this STH (12), to date, no plan exists for its control. Given the different transmission

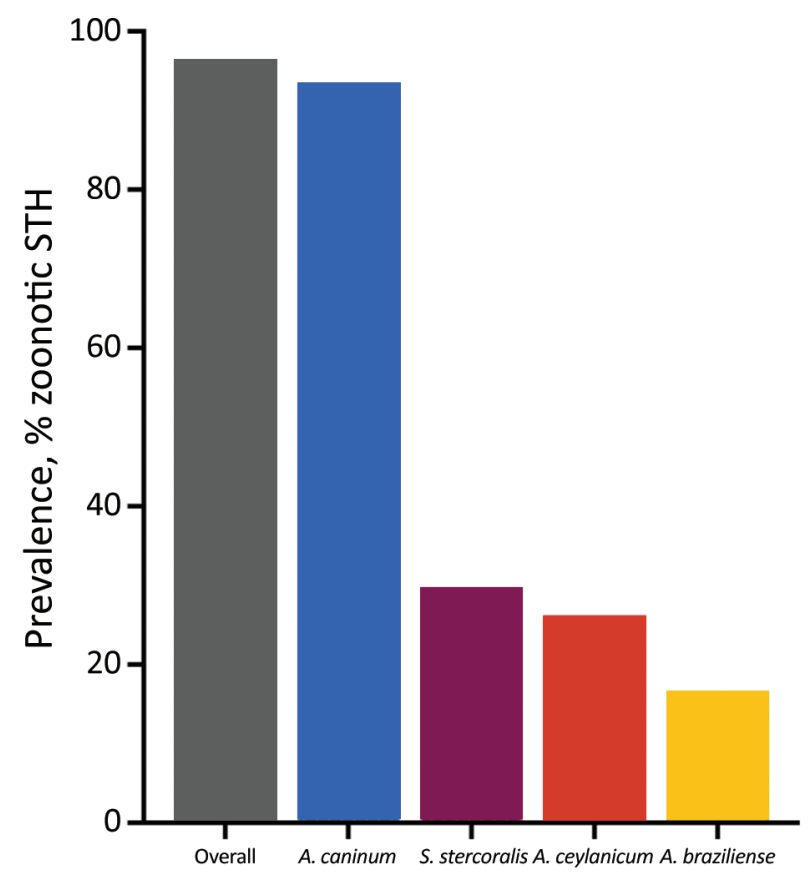

Figure 2. Prevalence of zoonotic soil-transmitted helminths in dogs on Tarawa Atoll, Kiribati. 
dynamics and infection outcomes with different zoonotic STHs, accurate identification of these parasites is essential for the implementation of effective therapy and control programs $(4,12)$. However, despite the efforts of nonprofit organizations, data on the occurrence of canine STHs in Kiribati were not previously available, hindering the understanding of the contribution of dogs in the transmission of zoonotic pathogens to humans.

Previous studies have shown an association between helminth infections and higher levels of anemia among school-age children from the Pacific region (13). Children with helminth infections were 3.6 times more likely to be stunted in growth and 2 times more likely to be anemic (13). This scenario is worsened by the absence of effective sewage systems, which contributes to the environmental contamination with animal and human feces, as demonstrated by the high levels of fecal coliforms in samples extracted from groundwater throughout South Tarawa (14). The lack of appropriate water, sanitation, and hygiene procedures increases the risks for infection with human and animal pathogens, including STHs. As a consequence, pneumonia and diarrhea, which have both strong links to hygiene and water, are some of the leading causes of illness and death among children in Kiribati (14).

In summary, we report a $96.5 \%$ prevalence of zoonotic STHs in dogs in Kiribati. Our results provide policy makers and key stakeholders with epidemiologic information that can be used for control programs to improve the health and quality of life of persons (in particular, women of reproductive age and children) and animals in the country, in line with the Kiribati-World Health Organization Cooperation Strategy 2018-2022.

\section{Acknowledgments}

We acknowledge the Ministry of Environment, Lands and Agriculture Developments, Republic of Kiribati, for their support during the implementation of this work. We also thank Leesa Grant, Annaliese Johnson, and Rosemary Miller for the sample collection and the FarrierVet/Mardi-Chi Dingo Foundation for leading the dog health and population management program.

\section{About the Author}

Ms. Zendejas-Heredia is a $\mathrm{PhD}$ candidate at the University of Melbourne, Melbourne, Australia. Her research is focused on the understanding of transmission dynamics of zoonotic soil-transmitted helminths in the Asia-Pacific region.

\section{References}

1. World Health Organization. Soil-transmitted helminth infections. Fact sheets. 2020 [cited 2021 Apr 21].

https://www.who.int/news-room/fact-sheets/detail/ soil-transmitted-helminth-infections

2. World Health Organization. 2030 targets for soil-transmitted helminthiases control programmes. 2020 [cited 2021 Apr 21]. https:/ / www.who.int/intestinal_worms/ resources/9789240000315/en/

3. Loukas A, Hotez PJ, Diemert D, Yazdanbakhsh M, McCarthy JS, Correa-Oliveira R, et al. Hookworm infection. Nat Rev Dis Primers. 2016;2:16088. https:/ / doi.org/10.1038/ nrdp. 2016.88

4. Massetti L, Colella V, Zendejas PA, Ng-Nguyen D, Harriott L, Marwedel L, et al. High-throughput multiplex qPCRs for the surveillance of zoonotic species of canine hookworms. PLoS Negl Trop Dis. 2020;14:e0008392. https://doi.org/10.1371/journal.pntd.0008392

5. Schär F, Inpankaew T, Traub RJ, Khieu V, Dalsgaard A, Chimnoi W, et al. The prevalence and diversity of intestinal parasitic infections in humans and domestic animals in a rural Cambodian village. Parasitol Int. 2014; 63:597-603. https://doi.org/10.1016/j.parint.2014.03.007

6. World Health Organization. Kiribati-WHO Country Cooperation Strategy 2018-2022. 2017 [cited 2021 Apr 21]. https://iris.wpro.who.int/handle/10665.1/13945

7. Storey D, Hunter S. Kiribati: An environmental "perfect storm." Aust Geogr. 2010;41:167-81. https://doi.org/ $10.1080 / 00049181003742294$

8. Verweij JJ, Canales M, Polman K, Ziem J, Brienen EAT, Polderman AM, et al. Molecular diagnosis of Strongyloides stercoralis in faecal samples using real-time PCR. Trans R Soc Trop Med Hyg. 2009;103:342-6. https:/ / doi.org/10.1016/ j.trstmh.2008.12.001

9. Montresor A, Cong DT, Sinuon M, Tsuyuoka R, Chanthavisouk C, Strandgaard H, et al. Large-scale preventive chemotherapy for the control of helminth infection in Western Pacific countries: six years later. PLoS Negl Trop Dis. 2008;2:e278. https://doi.org/10.1371/ journal.pntd.0000278

10. World Health Organization. Intestinal worms. 2020 [cited 2021 Apr 21]. https://www.who.int/intestinal_worms/ epidemiology/strongyloidiasis

11. Henriquez-Camacho C, Gotuzzo E, Echevarria J, White AC Jr, Terashima A, Samalvides F, et al. Ivermectin versus albendazole or thiabendazole for Strongyloides stercoralis infection. Cochrane Database Syst Rev. 2016;(1):CD007745. https://doi.org/10.1002/14651858.CD007745.pub3

12. Traub RJ. Ancylostoma ceylanicum, a re-emerging but neglected parasitic zoonosis. Int J Parasitol. 2013;43:1009-15. https:// doi.org/10.1016/j.ijpara.2013.07.006

13. Hughes RG, Sharp DS, Hughes MC, Akau'ola S, Heinsbroek P, Velayudhan R, et al. Environmental influences on helminthiasis and nutritional status among Pacific schoolchildren. Int J Environ Health Res. 2004;14:163-77. https://doi.org/10.1080/0960312042000218589

14. Government of Kiribati. Kiribati development plan 2016-19. 2016 [cited 2021 Apr 21]. http:/ / www.mfed.gov.ki/sites/ default/files/Kiribati\%20Development \%20Plan \%202016\%20 $-\% 2019 . p d f$

Address for correspondence: Vito Colella, Faculty of Veterinary and Agricultural Sciences, The University of Melbourne, Corner Flemington Rd and Park Dr, Parkville, VIC 3052, Australia; email: vito.colella@unimelb.edu.au 